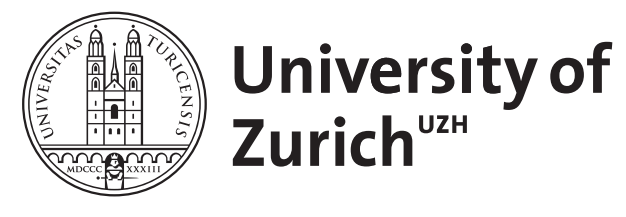

\title{
Nierensteine durch Migräne?
}

Gerber, L ; Wüthrich, R P ; Mohebbi, N

\begin{abstract}
A 42-year old woman was referred for a metabolic evaluation after two episodes of kidney stones. Her laboratory results revealed a normal anion-gap metabolic acidosis, a marked hypocitraturia $(0,6 \mathrm{mmol} / 24 \mathrm{~h}$; norm 1,6-4,5) and a urinary $\mathrm{pH}$ of 7,0 confirming renal tubular acidosis (RTA). We identified topiramate, our patient's medication for migraine, as the cause of the RTA. Topiramate, a carboanhydrase inhibitor leads to RTA of a mixed (proximal and distal) type and thus significantly increases the risk for kidney stones.
\end{abstract}

DOI: https://doi.org/10.1024/1661-8157/a000936

Other titles: Migraine-induced kidney stones?

Posted at the Zurich Open Repository and Archive, University of Zurich ZORA URL: https://doi.org/10.5167/uzh-70049

Journal Article

Originally published at:

Gerber, L; Wüthrich, R P; Mohebbi, N (2012). Nierensteine durch Migräne? Praxis, 101(10):665-668. DOI: https://doi.org/10.1024/1661-8157/a000936 


\author{
Klinik für Nephrologie, Universitätsspital Zürich \\ L. Gerber, R. P. Wüthrich, N. Mohebbi
}

\title{
Nierensteine durch Migräne?
}

Migraine-induced kidney stones?

Schlüsselwörter: Topiramat, renal tubuläre Azidose, Nephrolithiasis, Hypocitraturie Keywords: Topiramate, renal tubular acidosis, nephrolithiasis, hypocitraturia

Korrespondenzadresse:

Dr. med. Nilufar Mohebbi

Klinik für Nephrologie

Universitätsspital Zürich

Rämistrasse 100

8091 Zürich

E-mail: nilufar.mohebbi@usz.ch

Telefon:+41 442551111

Fax: +41 442554593 


\section{Zusammenfassung}

Eine 42 jährige Patientin wurde zur metabolischen Abklärung nach zwei Nierensteinenepisoden zugewiesen. Als Ursache zeigte sich eine renal tubuläre Azidose (RTA) bei einem Urin-pH von 7.0, metabolischer Azidose mit normaler Anionenlücke und deutlicher Hypocitraturie $(0.6 \mathrm{mmol} / 24 \mathrm{~h}$; Norm 1.6-4.5). Es stellte sich heraus, dass die RTA durch die Einnahme von Topiramat zur Rezidivprophylaxe bei schwerer Migräne bedingt war. Topiramat führt durch die strukturelle Ähnlichkeit mit Acetazolamid zu einer gemischten (proximalen und distalen) RTA und ist mit einer erhöhten Inzidenz von Nierensteinen assoziiert.

\section{Summary}

A 42-year old woman was referred for a metabolic evaluation after two episodes of kidney stones. Her laboratory results revealed a normal anion-gap metabolic acidosis, a marked hypocitraturia $(0.6 \mathrm{mmol} / 24 \mathrm{~h}$; norm $1.6-4.5)$ and a urinary $\mathrm{pH}$ of 7.0 confirming renal tubular acidosis (RTA). We identified topiramate, our patient's medication for migraine, as the cause of the RTA. Topiramate, a carboanhydrase inhibitor leads to RTA of a mixed (proximal and distal) type and thus significantly increases the risk for kidney stones. 


\section{Anamnese und Befunde}

Eine 42-jährige, aus Süditalien stammende, Patientin wurde zur Abklärung bei rezidivierenden Nierensteinen zugewiesen. Zwei Jahre zuvor war sie erstmals mit linksseitigen Flankenkoliken symptomatisch geworden, die im Verlauf ohne therapeutische Massnahmen vollständig regredient waren. Eineinhalb Jahre später hatten erneute kolikartige Schmerzen zum Nachweis von mehreren Konkrementen im linken Pyelon geführt, die mittels Ureterorenoskopie, Laserlithotrypsie und Steinextraktion vollständig entfernt werden konnten. Die Analyse eines Konkrementes mittels Röntgendiffraktion ergab eine Zusammensetzung aus $90 \%$ Apatit und $10 \%$ Calciumoxalatmonohydrat.

Bei der Erstvorstellung in unserer Steinsprechstunde war die Patientin beschwerdefrei. Die Systemanamnese war komplett unauffällig. Aufgrund einer chronischen Schizophrenie stand die Patientin seit mehreren Jahren unter einer Behandlung mit Risperidon. Zusätzlich bestand eine schwere Migräne seit vier Jahren, weshalb ca. 3 Jahre zuvor eine prophylaktische Therapie mit täglich $400 \mathrm{mg}$ Topiramat eingeleitet worden war. Die Patientin gab eine Flüssigkeitszufuhr von durchschnittlich $1.5 \mathrm{~L} / \mathrm{Tag}$ an, hauptsächlich in Form eines calciumarmen Mineralwassers. Oxalathaltige Nahrungsmittel wurden nur in geringen Mengen konsumiert. Die Zufuhr von Kochsalz und Eiweiss war moderat. Bezüglich Calciumzufuhr berichtete die Patientin, dass sie abgesehen von einem kleinen Stück Hartkäse (ca. einmal wöchentlich) keine Milchprodukte zu sich nahm. Die Familienanamnese ergab je eine Steinepisode bei einem Onkel väterlicherseits und bei einer Tante mütterlicherseits. Die klinische/körperliche Untersuchung war unauffällig.

Laborchemisch zeigte sich eine metabolische Azidose bei einem peripher venösen $\mathrm{pH}$ von 7.34 und einem erniedrigten Bicarbonat von $20.6 \mathrm{mmol} / \mathrm{l}$ (Norm 24mmol/l). Das Serumkalium betrug $3.6 \mathrm{mmol} / \mathrm{l}$. Die Anionenlücke im Serum war normal. Calcium, Phosphat, Kreatinin und intaktes Parathormon im Serum lagen im Normbereich. In der 24h-Urin-Sammlung zeigte sich eine deutliche Hypocitraturie $(0.60 \mathrm{mmol} / 24 \mathrm{~h}$; Norm 1.6 4.5) sowie eine eher hohe Kochsalzausscheidung (220 mmol/24h; Norm 40-220). Eine Hyperoxalurie, Hypercalciurie oder Hyperphosphaturie bestanden nicht. Der Urin-pH betrug 7.0, und im Urinsediment wurden Calciumphosphatkristalle nachgewiesen.

\section{Differentialdiagnostische Überlegungen}

Citrat ist der wichtigste protektive Faktor im Urin bei Nierensteinleiden. Citrat vermindert die Steinformation im Urin, in dem es freie Calciumionen bindet und somit eine Supersaturation mit Ausfällung von Calciumphosphat oder Calciumoxalat verhindert. 
Desweiteren übt Citrat einen direkten inhibitorischen Effekt auf das Wachstum von Calciumphosphatkristallen aus $[1,2]$. Bei niedrigem $\mathrm{pH}$ der proximalen Tubuluszellen wird vermehrt Citrat rückresorbiert, was zur Hypocitraturie führt und das Risiko für rezidivierende Nephrolithiasis entsprechend steigert. Die häufigste Ursache einer Hypocitraturie ist eine vermehrte Säurezufuhr in Form von tierischem Eiweiss in Kombination mit einem relativen Defizit an vegetarischen Produkten [1,2]. Die Eiweisszufuhr bei unserer Patientin war anamnestisch und aufgrund der gemessenen Eiweissumsatzrate (Protein Catabolic Rate (PCR)) von ca. $1 \mathrm{~g} / \mathrm{kg} / \mathrm{Tag}$ jedoch nicht exzessiv hoch. Weitere Ursachen für eine Hypocitraturie sind eine Hypokaliämie, eine Hypomagnesiämie, eine eingeschränkte Nierenfunktion und Harnwegsinfekte, wofür bei unserer Patientin kein Anhaltspunkt bestand [1].

Eine Hypocitraturie kann ebenfalls durch eine renal tubuläre Azidose (RTA) bedingt sein [1], wofür bei unserer Patientin mehrere Hinweise vorlagen. Sie wies eine hyperchlorämische metabolische Azidose mit normaler Anionenlücke auf, zeigte aber gleichzeitig einen inadäquat hohen Urin-pH (als Zeichen der Unfähigkeit der Niere Säure bzw. Säureäquivalente auszuscheiden) und entsprechend eine niedrige Nettosäureausscheidung von ca. $30 \mathrm{mmol} / 24 \mathrm{~h}$ (Norm bei einer typischen westlichen Diät ca. 40-80 mmol/24h). Die Nettosäureausscheidung wird bestimmt durch die Summe der Ammoniumausscheidung (ca. 2/3) und der ausgeschiedenen Menge an titrierbaren Säuren (ca. 1/3). Desweiteren war die Urin-Anionenlücke ((Natrium + Kalium) - Chlorid = ungemessene Anionen - ungemessene Kationen) positiv. Da die ungemessenen Kationen im Wesentlichen der Ammoniumausscheidung entsprechen, passte dieser Befund ebenfalls $\mathrm{zu}$ einer inadäquat niedrigen Säureausscheidung. In der Zusammenschau dieser Befunde stellten wir die Diagnose einer RTA.

Die renal tubulären Azidosen werden in 4 Typen unterteilt (distale RTA Typ 1, proximale RTA Typ 2, hyperkaliämische RTA Typ 4 und die gemischte RTA). Im Zusammenhang mit Nephrolithiasis von Bedeutung ist in erster Linie die distale RTA Typ 1 (syn. klassische RTA), die komplett oder inkomplett vorliegen kann. Typischerweise bedingt diese calciumphosphathaltige Nierensteine. Nebst angeborenen Formen (autosomal dominant oder rezessiv vererbte Mutationen, teilweise in Kombination mit neurosensorischer Schwerhörigkeit) finden sich zahlreiche erworbene Ursachen wie Autoimmunerkrankungen (z.B. Sjögren Syndrom, Rheumatoide Arthritis, Systemischer Lupus erythematodes), Erkrankungen, die mit Hypercalcämie bzw. Hypercalciurie assoziiert sind (z.B. primärer Hyperparathyreoidismus) oder andere Nierenerkrankungen, die mit einer tubulären Schädigung einhergehen wie z.B. Markschwammnieren, 
obstruktive Nephropathie oder Balkan-Nephropathie [3] (Tabelle 2). Auch Medikamente wie z.B. Lithium oder Amphotericin B können eine distale RTA verursachen [3]. Interessanterweise wurde in letzter Zeit mehrfach die Assoziation zwischen RTA und Topiramat, einem Medikament, das die Patientin aufgrund der Migräne in hoher Dosis einnahm, beschrieben [4].

Nach Ausschluss anderer Ursachen (z.B. Kollagenosen etc.) gingen wir somit davon aus, dass die Einnahme von Topiramat bei unserer Patientin die RTA und somit auch die rezidivierenden Steinepisoden verursacht hatte.

Da sie aufgrund der schweren und unter anderer Medikation therapieresistenten Migräne die Behandlung mit Topiramat nicht sistieren mochte, wurde versuchsweise eine Behandlung mit Kaliumcitrat und -hydrogencarbonat (Kalium Effervetten ${ }^{\circledR}$ ) eingeleitet. Daneben erfolgte eine Beratung hinsichtlich Steigerung der Trinkmenge auf mindestens 2-3 L/Tag sowie einer vermehrten Zufuhr von Früchten und Gemüse. Eine Osteodensitometrie ergab eine normale Knochendichte. Während den folgenden sechs Monaten kam zu keinen weiteren Steinepisoden mehr. Leider wurde die Medikation mit Kaliumcitrat von der Patientin nicht toleriert (Magenbeschwerden), so dass sie das Präparat wieder absetzte. Entsprechend zeigte sich, abgesehen von einem höheren Urinvolumen von jetzt $2.5 \mathrm{~L} / \mathrm{Tag}$ keine relevante Besserung des metabolischen Risikoprofils mit weiterhin nachweisbarer Hypocitraturie und neu einer Hypercalciurie. Letztere ist vermutlich durch ein aktuell höherer Kochsalzkonsum bedingt. Nebst erneuten diätetischen Instruktionen erfolgte die Umstellung von Kalium Effervetten ${ }^{\circledR}$ auf Kaliumcitrat Kapseln , die etwas besser toleriert wurden. Die Patientin wird aktuell in regelmässigen Abständen zur Verlaufskontrolle gesehen.

\section{Diagnose}

Topiramat-induzierte renal tubuläre Azidose mit rezidivierender Nephrolithiasis

\section{Kommentar}

Topiramat, 1996 von der FDA (Food and Drug Administration) ursprünglich als Antiepileptikum zugelassen, wurde in den letzten Jahren für eine zunehmende Anzahl von weiteren Indikationen eingesetzt (Bipolare Störungen, Migräne, Depression, Raucherentwöhnung, Posttraumatisches Stresssyndrom, diabetische Neuropathie), weshalb der Einsatz dieses Medikamentes in den letzten Jahren stark zugenommen hat. Bereits 2009 lag es unter den 40 umsatzstärksten Medikamenten in den USA [5]. Falls demnächst die Zulassung der FDA für eine neue „Schlankheitspille“, bestehend aus 
Topiramat und Phentermin erfolgt, dürfte die Zahl der mit Topiramat behandelten Patienten nochmals weiter ansteigen.

Neben diversen anderen pharmakologischen Wirkungen führt die Substanz aufgrund seiner strukturellen Ähnlichkeit $\mathrm{zu}$ Acetazolamid (ein häufig eingesetzter Carboanhydrasehemmer) zu einer Hemmung der Carboanhydrase Typ II und IV in den proximalen und distalen Tubuluszellen, was eine verminderte Bicarbonatreabsorption mit erhöhter fraktioneller Bicarbonatexkretion, Anstieg des Urin-pH und systemischer Azidose zur Folge hat [4]. Topiramat kann daher zu einer gemischten proximalen und distalen RTA führen. In einer Vergleichsstudie von 32 mit Topiramat behandelten Patienten und 50 gesunden Probanden konnte gezeigt werden, dass unter Topiramat der Urin-pH signifikant höher und die Citratexkretion signifikant niedriger war [4]. In einer anderen Arbeit konnte eine klare inverse Korrelation zwischen der Topiramatdosis und der Citraturie dokumentiert werden [6].

Topiramat führt aufgrund der metabolischen Azidose typischerweise auch zu einer Hypercalciurie, bedingt durch erhöhte Calciumresorption aus dem Knochen und Hemmung des Transient receptor potential channel type 5 (TRPC 5), ein Calciumkanal auf der luminalen Seite im distalen Tubulus [4]. Das initiale Fehlen der Hypercalciurie bei unserer Patientin führten wir am ehesten auf die geringe Calciumzufuhr und den Mangel an 25-OH-Vitamin D3 zurück. Im Verlauf nahm die Calciumexkretion deutlich zu.

Aufgrund dieser universellen Effekte auf die Urinzusammensetzung ist die in der Literatur genannte Prävalenz von Nierensteinen unter Topiramat erhöht. In einer retrospektiven Arbeit wurde bei 470 Patienten mit einer mittleren Behandlungsdauer von 13.8 Monaten eine Prävalenz für Nierensteine von $1.1 \%$ gefunden [4].

In Anbetracht des häufigen Gebrauchs von Topiramat in Zusammenhang mit dem erweiterten Indikationsspektrum und der erwarteten weiteren Zunahme der Verschreibungen in den nächsten Jahren ist das gehäufte Auftreten von Nierensteinen unter dieser Therapie von grosser klinischer Bedeutung. Die verschreibenden Ärzte der verschiedenen Disziplinen inklusive Allgemeinpraktiker und Internisten sollten diesen Zusammenhang kennen, die Patienten dahingehend informieren und mit Ihnen vorbeugende Massnahmen, insbesondere eine ausreichende Trinkmenge besprechen. Desweiteren sollten bei diesen Patienten während der Therapie regelmässige Kontrollen von Bicarbonat und Kalium durchgeführt werden. 


\section{Bibliographie}

1. Zuckerman JM, Assimons DG. Hypocitraturia: Pathophysiology and Medical Management. Rev Urol. 2009 Summer;11(3):134-44

2. Wagner CA, Mohebbi N. Urinary pH and stone formation. J Nephrol. 2010;23 Suppl 16:S165-9.

3. Ambühl PM. Pathophysiologie und Diagnostik renal tubulärer Azidosen. Ther Umsch. 2006;63(9):601-7.

4. Vega D, Maalouf NM, Sakhaee K. Increased propensity for calcium phosphate kidney stones with topiramate use. Expert Opin Drug Saf. 2007;6(5):547-57.

5. http://www.drugs.com/top200.html

6. Kaplon DM, Penniston KL, Nakada SY. Patients with and without prior urolithiasis have hypocitraturia and incident kidney stones while on topiramate. Urology. 2011;77(2):295-8. 